\section{Экспериментальное определение динамических свойств объектов регулирования}

\author{
Л. А. Нестеров ${ }^{1}$ \\ Петрозаводский государственный университет
}

\section{АННОТАЦИЯ}

В статье рассматривается метод исследования динамических свойств объектов автоматического регулирования путем снятия и обработки переходной характеристики.

Ключевые слова: объект, регулирование, емкость, самовыравнивание, запаздывание.

\section{SUMMARY}

In the article the method of research of dynamic properties of objects of automatic regulation is considered by measurement and processing of the transitive characteristics.

Keywords: object, regulation, capacity, self-alignment, delay.

Система автоматического регулирования - это замкнутая динамическая система, которая состоит из объекта регулирования и автоматического регулятора. В связи с этим качество регулирования зависит как от свойств регулятора, так и от свойств данного объекта.

Основными свойствами объекта регулирования являются емкость, самовыравнивание, запаздывание, время и скорость разгона.

В данной работе рассматривается определение динамических свойств объектов регулирования с помощью кривых разгона.

Емкость объекта. Емкостью регулируемого объекта называется запас накопленной энергии объектов или накопленного в объекте вещества. Одинаковые по величине возмущения по-разному влияют на регулируемую величину; чем больше емкость объекта регулирования, тем медленнее, при прочих равных условиях, будет изменяться регулируемая величина и, наоборот, чем меньше емкость объекта регулирования, тем он чувствительнее к этим возмущениям, т.е. объекты регулирования с большей емкостью более устойчивы. Влияние емкости объекта на регулируемую величину оценивается коэффициентом емкости.

Коэффициент емкости - то количество энергии или вещества, которое необходимо подвести в объект или отвести из объекта для того, чтобы изменить регулируемую величину на единицу измерения. Например,

\footnotetext{
${ }^{1}$ Автор - дочент кафедры промышленной теплотехники и энергосбережений

(С) Л. А. Нестеров, 2003
}

при регулировании температуры коэффициент емкости есть то количество тепла в ккал, которое необходимо подать, чтобы температура изменилась на $1^{\circ} \mathrm{C}$. Чем больше коэффициент емкости, тем больше емкость объекта, тем медленнее изменяется регулируемая величина, т.е. меньше его чувствительность к возмущениям, и наоборот.

В общем виде коэффициент емкости можно определить по формуле

$$
K_{c}=\frac{C}{R},
$$

где $C$ - емкость объекта регулирования;

$R$ - регулируемая величина.

Емкость каждого объекта разделяется на емкость со стороны потребления и со стороны подачи. Емкость со стороны потребления определяется характеристиками среды. Емкость со стороны подачи определяется характеристиками агента - вещества или энергии.

Процесс регулирования менее благоприятен, если объект имеет большую емкость со стороны подачи и малую - со стороны потребления, и благоприятен, когда емкости со стороны подачи меньше, чем со стороны потребления.

Самовыравнивание объекта. Самовыравниванием называется свойство регулируемого объекта после возмущения стремиться вновь прийти в состояние равновесия без внешнего воздействия. Объекты без самовыравнивания характеризуются тем, что при нарушении равновесия за счет отклонения регулируемой величины равновесие не восстанавливается. Такие объекты практически не поддаются регулированию.

Самовыравнивание может быть положительным и отрицательным. Объекты с положительным самовыравниванием характеризуется тем, что при изменении регулируемой величины нарушенное равновесие восстанавливается без участия регулятора, а с отрицательным самовыравниванием нарушенное равновесие может быть восстановлено с помощью регулятора.

Способность объекта регулирования к самовыравниванию характеризуется коэффициентом самовыравнивания

$$
\rho=\frac{d q}{d \sigma},
$$

где $q$ - разность притока и расхода в объекте;

$\sigma$ - отклонение регулируемой величины в объекте.

Коэффициент самовыравнивания численно равен отношению величины возмущающегося воздействия к отклонению регулируемой величины, вызванному этим воздействием.

Самовыравнивание способствует устойчивости регулируемого объекта; чем больше коэффициент самовы- 
равнивания, тем быстрей объект самостоятельно восстанавливает заданное значение регулируемой величины и более устойчивым будет процесс регулирования.

Коэффициент самовыравнивания не является постоянной величиной, т.к. зависит от нагрузки на объект; чем меньше нагрузка, тем меньше коэффициент и тем труднее обеспечить устойчивое и качественное регулирование.

Запаздывание процесса в объекте. Изменение регулируемой величины с момента возмущения происходит не сразу, а через некоторое время, которое называется запаздыванием процесса в объекте. Запаздывание присуще не только регулируемому объекту, но и регулятору и зависит от инерционности чувствительного элемента, кинематики привода регулирующего органа и системы передачи командного сигнала.

К свойствам объекта регулирования относится запаздывание передаточное и переходное.

Передаточное запаздывание - это время, в течение которого регулируемая величина, несмотря на возмущение, не изменяется. Передаточное запаздывание зависит от нагрузки объекта. Чем больше нагрузка, тем меньше передаточное запаздывание, т.к. при большой нагрузке регулируемая среда движется быстрее и чувствительный элемент будет реагировать на это возмущение раньше. Передаточное запаздывание зависит от емкости объекта: чем больше емкость, тем больше время передаточного запаздывания. Для уменьшения времени передаточного запаздывания регулирующий орган необходимо располагать ближе к объекту, чтобы емкость между ними была наименьшей.

Переходным запаздыванием процесса регулирования называется запаздывание, зависящее от тепловых, гидравлических и других сопротивлений между емкостями объекта, и определяется как промежуток времени от момента возмущения до начала изменения регулируемой величины.

Сумма времени передаточного и переходного запаздываний называется временем полного запаздывания:

$$
\tau=\tau_{0}+\tau_{n},
$$

где $\tau_{0}$ - время передаточного запаздывания;

$$
\tau_{n} \text { - время переходного запаздывания. }
$$

Запаздывание существенно влияет на величину динамической ошибки: чем больше запаздывание, тем больше ошибка

Время разгона объекта. Временем разгона объекта является время, в течение которого регулируемая величина изменяется от нуля до заданного значения при мгновенном возмущении при условии, что скорость изменения величины остается постоянной, а нагрузка отсутствует. Чем больше емкость объекта регулирования, тем больше время разгона.

Время разгона определяется уравнением:

$$
T=\frac{\Delta q d t}{d \sigma},
$$

где $\Delta q$ - относительная величина возмущения;

$d t$ - изменение времени;

$d \sigma$ - изменение регулируемой величины.

Величина, обратная времени разгона, называется скоростью разгона. Чем больше возмущающее воздействие, тем быстрее изменяется регулируемая величина, т.е. тем больше будет скорость этого измерения. Если при установившемся режиме объекта отключить регулятор и резко увеличить нагрузку, то в зависимости от указанных выше свойств объекта можно записать изменение регулируемой величины во времени. Эта зависимость называется переходной характеристикой или кривой разгона объектов регулирования (рис. 1).

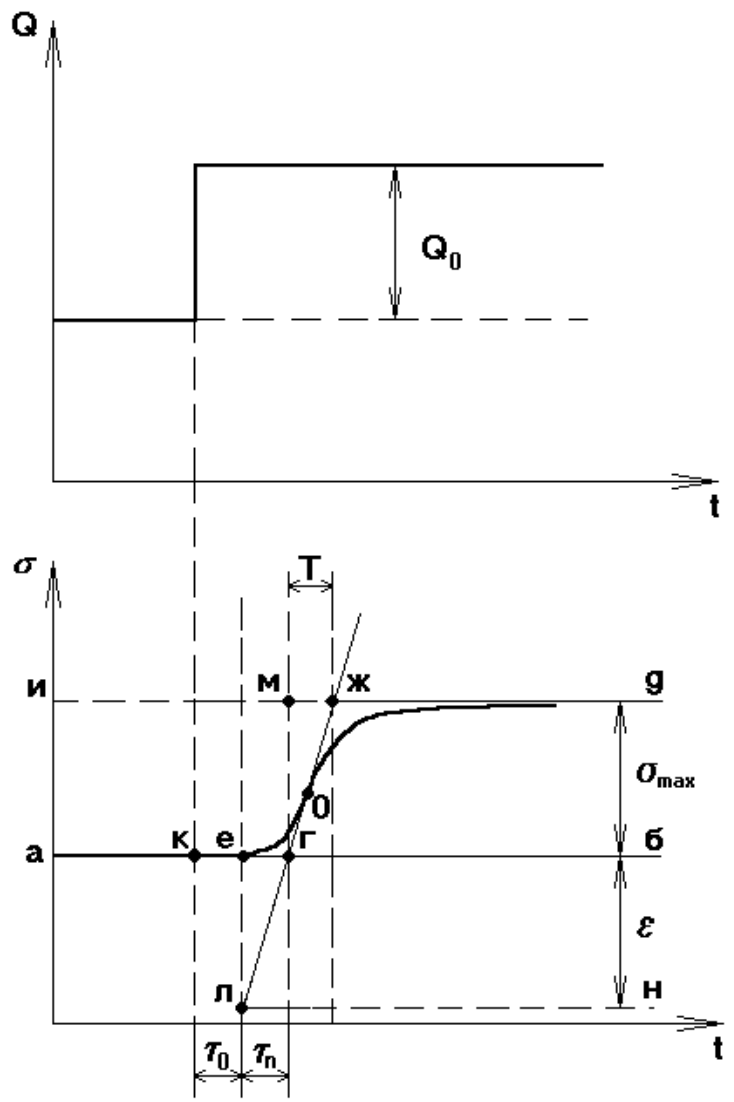

Рис. 1. Кривая разгона для определения динамических свойств объектов регулирования

После анализа и обработки кривой разгона можно определить время запаздывания объекта, коэффициент самовыравнивания, изменение регулируемой величи- 
ны после возмущения, постоянную времени объекта и влияние нагрузки на регулируемую величину.

1. Время передаточного запаздывания определяют от начала возмущения до момента изменения регулируемой величины. Отрезок ке в масштабе есть передаточное запаздывание $\tau_{0}$.

Для определения времени переходного запаздывания проводят линию начального значения регулируемой величины $а б$, находят точку максимальной скорости ее изменения (точка перегиба $O)$ и через нее проводят касательную до пересечения с линией аб. Полученный отрезок ег в масштабе есть переходное запаздывание $\tau_{n}$.

2. Для определения времени разгона $T$ проводят линию $и д$ (новое установившееся значение регулируемой величины), проводят касательную до пересечения ее с линией $и \partial$ в точке $ж$, проводят вертикальную линию через точку г до точки $\mathcal{M}$. Полученный отрезок мж в масштабе есть время разгона объекта или его постоянная времени. Эта величина характеризует продолжительность процесса самовыравнивания и характер изменения регулируемой величины во времени. Для одноемкостного объекта с момента возмущения (точка $c$ ) регулируемая величина достигнет точки $O$ и составит $63,2 \%$ от предельного отклонения $\tau_{\max }$. Через время $2 T \tau$ достигнет значения $0,856 \tau_{\max }$, через $3 T-0,956 \tau_{\max }$, т.е. практически процесс самовыравнивания закончится.

3. Для определения коэффициента самовыравнивания определяют по кривой разгона $\sigma_{\max }$. По величине изменения нагрузки $Q_{0}$ определяют коэффициент самовыравнивания

$$
\rho=\frac{Q_{0}}{\sigma_{\max }} .
$$

По коэффициенту самовыравнивания можно определить коэффициент усиления объекта, т.к.

$$
K=\frac{1}{\rho} .
$$

Величина, обратная времени разгона, является скоростью разгона, которая также может быть определена по кривой разгона. Для этого необходимо через точку $e$ провести вертикальную линию до пересечения касательной в точке $л$ и провести через эту точку линию $л н$, параллельно линии аб. Полученный отрезок $\varepsilon$ и есть скорость разгона объекта, т.е. максимальная скорость изменения регулируемой величины в процессе самовыравнивания.

Анализ и обработка кривой разгона объекта регулирования позволяют определить основные показатели, которые определяют его динамические свойства. На основании этих показателей производится выбор регулятора и корректирующих звеньев, обеспечивающих устойчивость и высокое качество процесса регулирования.

\section{СПИСОК ЛИТЕРАТУРЫ}

1. Клюев А. С. Автоматическое регулирование. М.: Энергия, 1973. $391 \mathrm{c}$.

2. Балмасов Е. Я. Автоматизация процессов производства деревянных плит. М.: Лесная промышленность, $1973.223 \mathrm{c}$.

3. Нестеров Л. А. Определение динамических характеристик объектов регулирования по кривым разгона / Петрозаводский гос. ун-т. Петрозаводск, 2001. 6 с . Деп. в ВИНИТИ 12.03.01, №619-В 2001. 